\title{
Genome-wide association study of platelet aggregation in African Americans
}

\author{
Rehan Qayyum ${ }^{1,2^{*}}$, Lewis C. Becker ${ }^{1}$, Diane M. Becker ${ }^{1}$, Nauder Faraday ${ }^{3}$, Lisa R. Yanek' ${ }^{1}$, Suzanne M. Leal ${ }^{4}$, \\ Chad Shaw ${ }^{4}$, Rasika Mathias ${ }^{1}$, Bhoom Suktitipat ${ }^{5}$ and Paul F. Bray ${ }^{6}$
}

\begin{abstract}
Background: We have previously shown that platelet aggregation has higher heritability in African Americans than European Americans. However, a genome-wide association study (GWAS) of platelet aggregation in African Americans has not been reported. We measured platelet aggregation in response to arachidonic acid, ADP, collagen, or epinephrine by optical aggregometry. The discovery cohort was 825 African Americans from the GeneSTAR study. Two replication cohorts were used: 119 African Americans from the Platelet Genes and Physiology Study and 1221 European Americans from GeneSTAR. Genotyping was conducted with Illumina $1 \mathrm{M}$ arrays. For each cohort, age- and sex-adjusted linear mixed models were used to test for association between each SNP and each phenotype under an additive model.

Results: Six SNPs were significantly associated with platelet aggregation $\left(P<5 \times 10^{-8}\right)$ in the discovery sample. Of these, three SNPs in three different loci were confirmed: 1) rs12041331, in PEAR1 (platelet endothelial aggregation receptor 1), replicated in both African and European Americans for collagen- and epinephrine-induced aggregation, and in European Americans for ADP-induced aggregation; 2) rs11202221, in BMPR1A (bone morphogenetic protein receptor type1A), replicated in African Americans for ADP-induced aggregation; and 3) rs6566765 replicated in European Americans for ADP-induced aggregation. The rs11202221 and rs6566765 associations with agonist-induced platelet aggregation are novel.
\end{abstract}

Conclusions: In this first GWAS of agonist-induced platelet aggregation in African Americans, we discovered and replicated, novel associations of two variants with ADP-induced aggregation, and confirmed the association of a PEAR1 variant with multi-agonist-induced aggregation. Further study of these genes may provide novel insights into platelet biology.

Keywords: Platelet aggregation, PEAR1, BMPR1A, Genome-wide association study

\section{Background}

Human blood platelets play an essential role in normal hemostasis and in pathologic thrombosis, in particular arterial thrombosis [1]. There is accumulating evidence that platelets also participate in the development, progression, and manifestations of atherosclerotic diseases [2]. Studies of ex vivo agonist-induced platelet aggregation have shown large and reproducible variations among individuals [3]. In patients receiving anti-platelet therapy for secondary cardiovascular prevention, greater platelet aggregability ex vivo is associated with increased

\footnotetext{
* Correspondence: rqayyum@jhmi.edu

${ }^{1}$ The GeneSTAR Research Center, Department of Medicine, Johns Hopkins University School of Medicine, Baltimore, Maryland 21287, USA ${ }^{2}$ Department of Medicine, University of Tennessee College of Medicine, Chattanooga, Tennessee 37403, USA

Full list of author information is available at the end of the article
}

risk of cardiovascular disease events $[4,5]$. Several cardiovascular risk factors are known to affect platelet aggregation in healthy individuals including age, sex, obesity, and the presence of metabolic syndrome [6-10]. The majority of this variation in platelet aggregation is heritable. Using a family study design, we have previously reported that greater than $70 \%$ of variation in platelet aggregation in African Americans and almost $60 \%$ of variation in European Americans is heritable [11].

Several candidate gene studies have examined the association of genetic variants in specific genes with platelet aggregation with inconsistent results [12]. A genome-wide association study in European Americans identified seven loci associated with agonist-induced platelet aggregation [13]. However, a genome-wide association study of platelet aggregation in African Americans has not yet been 
reported. The genetic variants reported to date explain only a small fraction of the heritability in platelet aggregation, providing an opportunity for new studies to discover additional genetic variants of importance. Moreover, African Americans have higher heritability of platelet aggregation than European Americans and additional genetic variants may contribute to this difference [11]. Because of the different allele frequencies and linkage disequilibrium patterns in populations of European and African ancestry, we anticipated that we might discover new genetic loci associated with platelet aggregation in an African American population compared to European Americans [14]. Therefore, we performed a genome-wide association study (GWAS) in African Americans to identify genetic variants that determine agonist-induced platelet aggregation and replicated our findings in independent samples of African Americans and European Americans $[4,5]$.

\section{Results}

The discovery sample from GeneSTAR consisted of 825 African Americans. The replication samples consisted of 119 African Americans in PGAP and 1221 European Americans in GeneSTAR (Table 1). Participants from the GeneSTAR cohorts were older with greater percentages of hypertensives and smokers. They also had higher platelet count and fibrinogen levels. Overall, 802,881 genotyped SNPs passed our quality control criteria in all studies and were included in analyses. Table 2 shows the gene variants that were significantly associated with agonist-induced platelet aggregation at the GWAS level in African American GeneSTAR participants. The quantile-quantile plots are presented in Additional file 1: Fig. S1 with inflation of test statistics (lambda range 1.04 to 1.08$)$.

Table 1 Study population characteristics

\begin{tabular}{llll}
\hline Characteristics & $\begin{array}{l}\text { GeneSTAR } \\
(\mathrm{AA})(\mathrm{N}=825)\end{array}$ & $\begin{array}{l}\text { PGAP (AA) } \\
(\mathrm{N}=119)\end{array}$ & $\begin{array}{l}\text { GeneSTAR } \\
(\mathrm{EA})(\mathrm{N}=1221)\end{array}$ \\
\hline Age, years & $45(12)$ & $35(9)$ & $44(13)$ \\
Female & $62 \%$ & $72 \%$ & $55 \%$ \\
Hypertension & $39 \%$ & $8 \%$ & $26 \%$ \\
Smoker & $30 \%$ & $14 \%$ & $23 \%$ \\
Body Mass Index, Kg/m & $32(8)$ & $29(6)$ & $28(6)$ \\
Fibrinogen, $\mathrm{mg} / \mathrm{dL}$ & $375(111)$ & $349(101)$ & $374(111)$ \\
Platelet Count, $10^{9} / \mathrm{L}$ & $266(68)$ & $236(53)$ & $261(62)$ \\
Mean Platelet Volume, $10^{-15} \mathrm{~L}$ & $8(1)$ & $7(1)$ & $7(1)$ \\
Von Willebrand Factor, \% & $86(53)$ & $87(38)$ & $87(58)$
\end{tabular}

Data is presented as mean (standard deviation) unless noted otherwise Abbreviations: EA European Americans, AA African Americans Population Characteristics of the GeneSTAR (Genetic Study of Atherosclerosis Risk) and PGAP (Platelets Genes and Physiology) cohorts

\section{Epinephrine-induced platelet aggregation}

The A-allele of rs12041331, located in the first intron of PEAR1, was associated with decreased platelet aggregation to $2 \mu \mathrm{M}$ epinephrine $\left(\mathrm{p}=8.34 \times 10^{-12}\right)$ (Fig. 1 and Additional file 1 Table S1). The association of this SNP has been previously reported by our group and the Framingham Heart Study with platelet aggregation phenotypes in both European Americans and African Americans $[13,15]$. Both replication cohorts validated the association of this SNP with epinephrine-induced platelet aggregation (African Americans: $\mathrm{p}=4.6 \times 10^{-3}$; European Americans: $\mathrm{p}=4.47 \times 10^{-3}$ ) with a similar direction of effect. Suggestive association was noted with SNPs at 6 loci but none were significant in the replication samples (Additional file 1: Table S2).

\section{Collagen-induced platelet aggregation}

The PEAR1 SNP rs12041331 was also associated with platelet aggregation to $2 \mu \mathrm{g} / \mathrm{mL}$ collagen (Fig. $1 ; \mathrm{p}=2.7 \times$ $\left.10^{-11}\right)$. This association was also present in both replication cohorts (African American: $\mathrm{p}=9.0 \times 10^{-3}$; European American: $\mathrm{p}=0.01$ ) although $\mathrm{p}$-values did not cross Bonferroni-adjusted p-value threshold. The minor allele, A, was associated with lesser platelet aggregation in all cohorts. In addition, 6 SNPs had suggestive significant association with aggregation to with $2 \mu \mathrm{g} / \mathrm{mL}$ collagen (Additional file 1: Table S3).

\section{ADP-induced platelet aggregation}

The PEAR1 SNP rs12041331 was also significantly associated with platelet aggregation after stimulation with both doses of ADP $\left(p=5.8 \times 10^{-9}\right.$ for $2 \mu \mathrm{M}$ ADP and $\mathrm{p}=3.2 \times$ $10^{-10}$ for $10 \mu \mathrm{M}$ ADP) (Fig. 1). Three additional SNPs were significantly associated with platelet aggregation after stimulation with $2 \mu \mathrm{M}$ ADP. One SNP, rs11924165, was in the non-protein coding region of ALDH1L1-AS2 (aldehyde dehydrogenase 1 family, member L1-antisense RNA 2 ); the minor allele was associated with increased platelet aggregation $\left(\mathrm{P}=1.17 \times 10^{-8}\right)$. The second SNP, rs10883735, was located in the $2^{\text {nd }}$ intron of SUFU gene $(\mathrm{p}=2.2 \times$ $10^{-8}$ ). The third SNP, rs11202221, was located in the 2nd intron of the BMPR1A gene $\left(\mathrm{p}=4.8 \times 10^{-8}\right)$. The minor allele of this SNP was associated with decreased platelet aggregation (Additional file 1: Table S1 and S4). Five additional SNPs in BMPR1A had suggestive associations with p-values $<5 \times 10^{-7}$ (Fig. 2; Additional file 1: Table S5); all five SNPs were in strong LD with the lead SNP, rs11202221 of BMPR1A (Fig. 2).

Two additional SNPs were significantly associated with platelet aggregation after stimulation with $10 \mu \mathrm{M}$ ADP (Table 2). One SNP, rs6566765, was located in an intergenic region with no known protein-coding gene within the flanking $250 \mathrm{~kb}$ and its minor allele was associated with increased platelet aggregation (Additional file 1: 
Table 2 Genome-wide association study results

\begin{tabular}{|c|c|c|c|c|c|c|c|c|c|c|c|}
\hline \multirow[b]{2}{*}{ SNP_CA } & \multirow[b]{2}{*}{ Position } & \multirow[b]{2}{*}{ Gene } & \multicolumn{3}{|c|}{ GeneSTAR (AA) } & \multicolumn{3}{|l|}{ PGAP } & \multicolumn{3}{|c|}{ GeneSTAR (EA) } \\
\hline & & & $\beta(\mathrm{SE})$ & P-value & MAF & $\beta(\mathrm{SE})$ & P-value & MAF & $\beta(\mathrm{SE})$ & P-value & MAF \\
\hline \multicolumn{12}{|c|}{ Epinephrine $2 \mu \mathrm{M}$} \\
\hline rs12041331_A & 1:155136338 & PEAR1 & $-0.93(0.13)$ & $2.82 \times 10-{ }^{12}$ & $29.2 \%$ & $-0.19(0.05)$ & $4.64 \times 10^{-3}$ & $32.8 \%$ & $-0.47(0.18)$ & $4.47 \times 10^{-3}$ & $9.1 \%$ \\
\hline \multicolumn{12}{|c|}{ Collagen $2 \mu \mathrm{g} / \mathrm{mL}$} \\
\hline rs12041331_A & 1:155136338 & PEAR1 & $-0.89(0.13)$ & $2.74 \times 10^{-11}$ & $35.8 \%$ & $-1.55(0.59)$ & $9.0 \times 10^{-3}$ & $32.1 \%$ & $-0.47(0.18)$ & 0.01 & $9.1 \%$ \\
\hline \multicolumn{12}{|l|}{ ADP $2 \mu \mathrm{M}$} \\
\hline rs12041331_A & 1:155136338 & PEAR1 & $-9.2(1.56)$ & $5.8 \times 10^{-9}$ & $35.8 \%$ & $-3.65(2.41)$ & 0.13 & $33.2 \%$ & $-8.52(1.87)$ & $6.08 \times 10^{-6}$ & $9.1 \%$ \\
\hline rs11924165_T & 3:127391893 & ALDH1L1-AS2 & $9.72(1.68)$ & $1.17 \times 10^{-8}$ & $16.9 \%$ & $-5.10(2.86)$ & 0.07 & $20.0 \%$ & - & - & $-*$ \\
\hline rs10883735_T & 10:104298436 & SUFU & $-13.9(2.45)$ & $2.18 \times 10^{-8}$ & $5.1 \%$ & $1.72(4.28)$ & 0.69 & $8.3 \%$ & $1.36(2.45)$ & 0.48 & $8.1 \%$ \\
\hline rs11202221_G & 10:88592294 & BMPR1A & $-12.72(2.30)$ & $4.8 \times 10^{-8}$ & $7.3 \%$ & $-16.04(4.06)$ & $7.71 \times 10^{-5}$ & $9.1 \%$ & $0.60(1.50)$ & 0.69 & $20.5 \%$ \\
\hline \multicolumn{12}{|l|}{ ADP $10 \mu \mathrm{M}$} \\
\hline rs12041331_A & 1:155136338 & PEAR1 & $-5.63(0.88)$ & $3.2 \times 10^{-10}$ & $35.8 \%$ & $-3.65(2.41)$ & 0.13 & $32.1 \%$ & $-2.57(1.36)$ & 0.06 & $9.1 \%$ \\
\hline rs6566765_T & 18:69569776 & & $4.72(0.84)$ & $3.59 \times 10^{-8}$ & $39.7 \%$ & $1.78(2.49)$ & 0.34 & $39.3 \%$ & $2.01(0.62)$ & $1.3 \times 10^{-3}$ & $35.1 \%$ \\
\hline rs9889955_G & 17:69072972 & SDK2 & $-6.42(1.15)$ & $4.14 \times 10^{-8}$ & $23.5 \%$ & $-0.47(2.71)$ & 0.86 & $25.9 \%$ & $-0.32(0.74)$ & 0.66 & $39.3 \% \dagger$ \\
\hline
\end{tabular}

Abbreviations: SNP single nucleotide polymorphism, CA coded allele, $\beta$ regression coefficient, SE standard error, GeneSTAR Genetic Study of Atherosclerosis, PGAP Platelet Gene and Physiology, MAF minor allele frequency, $A D P$ adenosine diphosphate, $A A$ African Americans, EA European Americans

Note: In PGAP the epinephrine concentration of $1.5 \mathrm{uM}$, ADP concentration of $4 \mathrm{uM}$, and collagen concentration of $2.5 \mathrm{ug} / \mathrm{mL}$ were used

* This SNP is monomorphic in population of European descent. Another SNP, rs7611945_A, 231,689 bp upstream of rs 11924165 was statistically significant $\left(\beta(S E)=-4.68(1.32) ; p=4.25 \times 10^{-4} ;\right.$ MAF $\left.=20 \%\right)$ after adjusting for the

number of SNPs in between $(N=97)$ or the number of $L D$ blocks $(N=20)$

† Another variant in SDK2 gene, rs11869008_G, located 140,506 bp downstream of rs9889955 was statistically significant ( $\left.\beta(S E)=-2.79(0.84) ; P=9.66 \times 10^{-4} ; \mathrm{MAF}=19.7 \%\right)$ after adjusting for the number of LD blocks in YRI ( $N=25$ ) but not after adjusting for the number of genotyped SNPs $(\mathrm{N}=63)$

Genome-wide association study results of platelet aggregation in discovery cohort of African Americans and replication cohorts of African Americans and European Americans 


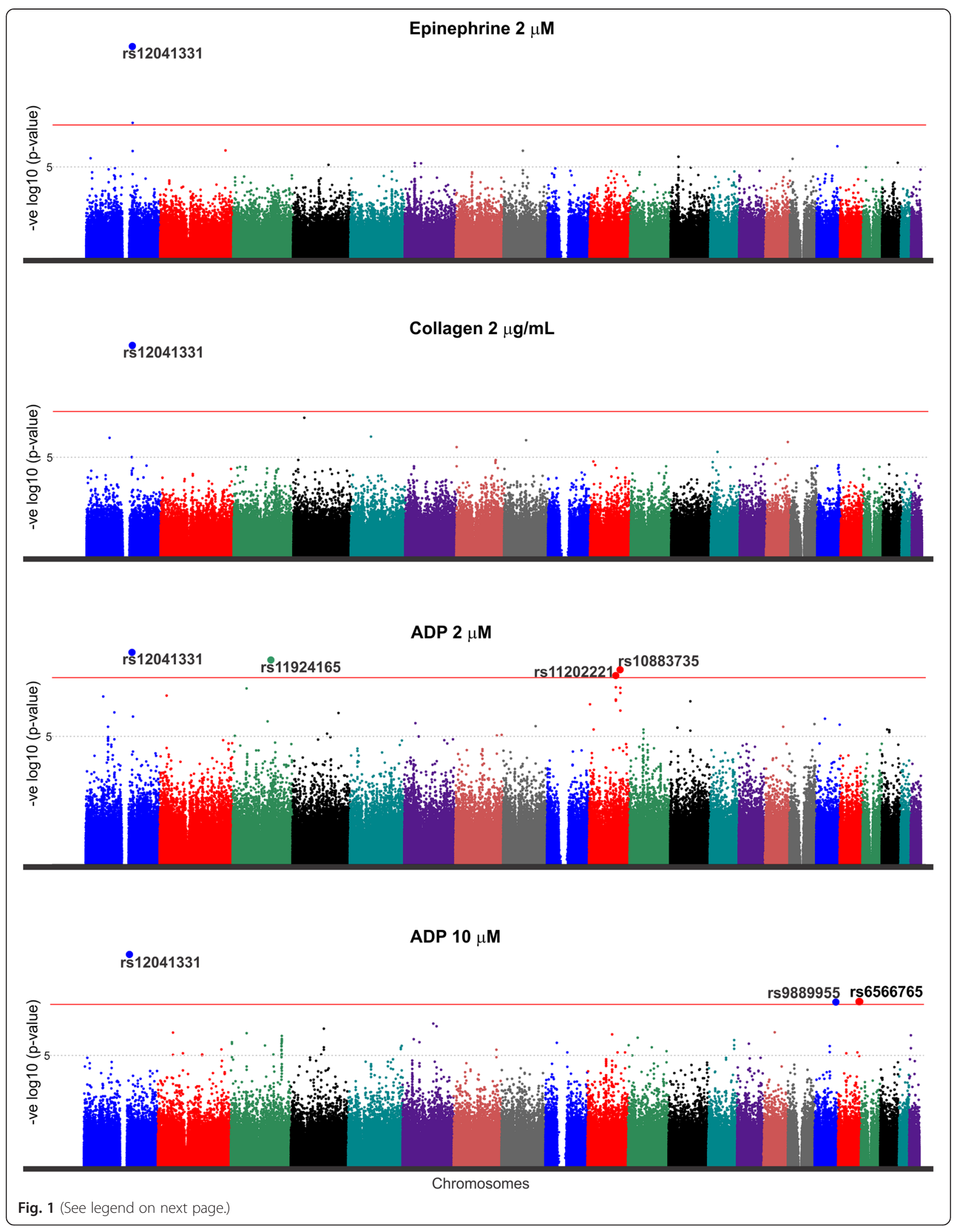


(See figure on previous page.)

Fig. 1 Manhattan plot of the genome-wide association results of agonist-mediated platelet aggregation. The $y$-axis represents the negative logarithm (base 10) of p-values and the $x$-axis represents chromosomes with positions of genetic variants. The horizontal red line represents the genome-wide significance threshold. Results of arachidonic acid-mediated platelet aggregation are not shown here as no genetic variant crossed genome-wide significance threshold

Table S1 and S4). Another SNP, rs9889955, was located in the first intron of $S D K 2$.

Of the six SNPs associated with ADP-induced platelet aggregation in the discovery sample, only the $\mathrm{G}$ allele of the rs11202221 in BMPR1A was associated with decreased ADP-induced platelet aggregation in the African American replication sample $\left(\mathrm{p}=9.17 \times 10^{-5}\right.$; Table 2$)$. Furthermore, the 5 SNPs with suggestive significance in $B M P R 1 A$ in the discovery sample were also significant in the African American replication sample (Fig. 2; all pvalues $<9 \times 10^{-5}$ ). However, neither rs11202221 nor any nearby SNP was associated with ADP-induced aggregation in the European American replication cohort, likely due to differences in linkage disequilibrium between the two populations (Additional file 1: Fig. S3).

The A allele of rs12041331 in PEAR1 was not significantly associated with platelet aggregation in the PGAP replication sample, although the direction of effect was

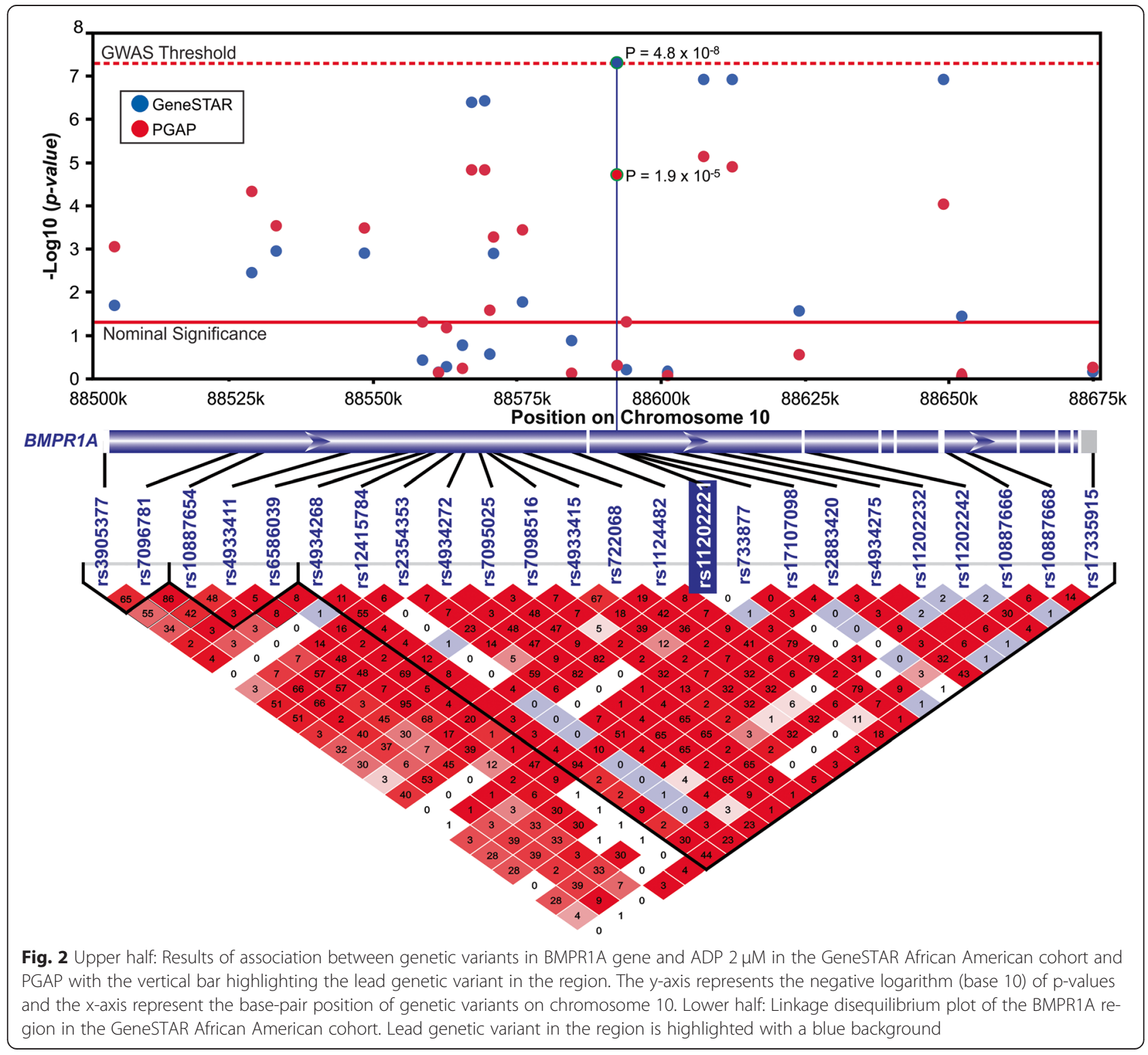


similar for both concentrations of ADP $(p=0.14)$. On the other hand, this SNP was associated with decreased platelet aggregation after stimulation with $2 \mu \mathrm{M}$ ADP in the European American cohort and had a similar direction of effect $\left(\beta[\mathrm{SE}]=-9.2[1.56], \mathrm{p}=5.8 \times 10^{-9}\right)$. The $\mathrm{T}$ allele of the intergenic variant, rs6566765, was associated with increased platelet aggregation to $10 \mu \mathrm{M}$ ADP in the European American cohort $(\beta[\mathrm{SE}]=2.01[0.62] ; \mathrm{p}=1.3 \times$ $10^{-3}$ ) but not in the African American replication cohort. When ADP-mediated platelet aggregation with both doses was examined across the genotypes of rs11202221 and rs6566765, we found that increasing allele dose (G-allele for rs11202221 and C-allele for rs6566765) was associated with decreased platelet aggregation (Additional file 1: Table S4). The rs9889955 variant in SDK2 was not associated with platelet aggregation in either of the two replication cohorts.

Suggestive associations between platelet aggregation induced by $2 \mu \mathrm{M}$ ADP and $10 \mu \mathrm{M}$ ADP were noted for SNPs at 16 loci and 23 loci, respectively, but only 1 of these SNPs was replicated (Additional file 1: Tables S5 and S6). The minor allele of the replicated variant, rs750693 in FRMPD1, was also associated with an increase in platelet aggregation in response to $10 \mathrm{uM} \mathrm{ADP}$ in the European American replication sample.

\section{Arachidonic acid-induced platelet aggregation}

We did not find any locus that crossed GWAS threshold for arachidonic acid-mediated platelet aggregation in the discovery sample. We found 10 loci that had suggestive association with arachidonic acid-induced platelet aggregation (Additional file 1: Table S7), but none were nominally associated with arachidonic acid-induced aggregation in the replication samples.

\section{Discussion}

Lower coronary artery disease survival rates have been observed in African Americans even after clinical, demographic and socioeconomic variables are considered $[16,17]$, suggesting there are undiscovered factors accounting for this racial difference. We and others have shown there is a strong genetic component to coronary artery disease and platelet reactivity $[11,18-20]$, but there is a paucity of data about responsible genetic mechanisms. Thus, the African American participants in the GeneSTAR and PGAP studies represent unique and valuable resources for discovering novel genetic variants associated with platelet aggregation, a central process in acute coronary syndromes. We used the large GeneSTAR African American cohort as the GWAS discovery sample, and separate PGAP and GeneSTAR European Americans as replication cohorts. The major findings were: 1 ) identification of 3 replicated variants associated with platelet aggregation in the discovery sample, 2) rs12041331 in
PEAR1 (previously reported genetic variant in European Americans and African Americans), was associated with collagen, epinephrine and ADP aggregation in both the discovery and replication cohorts, 3) rs11202221 in BMPR1A was associated with ADP aggregation in both the discovery and the African American replication cohort, and 4) rs6566765 was associated with ADP aggregation in the African American discovery and European American replication cohort. Since African Americans are under-represented in most clinical studies of coronary artery disease (CAD), it will be important to consider these established and novel genetic variants in this racial group.

Despite modest sample sizes of the discovery and replication cohorts, we were able to identify and validate three loci associated with platelet aggregation. The finding of significant loci in modestly sized cohorts is not typical of most GWAS studies. We had large effect sizes for these loci, probably because platelet aggregation is more physiologically defined than most clinical phenotypes and represents a biological process, not a disease outcome. The large effect sizes are likely due to the relatively large percentage of heritability explained by the discovered loci in African Americans (from $10 \%$ with ADP $2 \mathrm{uM}$ to $17 \%$ with epinephrine). With a larger sample size, we probably would have discovered additional genetic variants with smaller effect sizes.

Assessment of ex vivo platelet function is labor intensive and very few cohorts have been generated with this phenotype; fewer still have substantial numbers of African Americans. In general, compared to the PGAP cohort, GeneSTAR participants had a higher incidence of CAD risk factors. Nevertheless, the minor allele frequencies (MAF) for each of the African American cohorts in this report were remarkably similar (Table 2). The GeneSTAR and PGAP studies also utilized the same platelet agonists and same genotyping platform, features of the study design that support the validity of our analyses. The replicated variants were not associated with fibrinogen levels in any cohort (Additional file 1: Table S1).

The rs12041331 SNP in PEAR1 has previously been associated with epinephrine-and ADP-mediated platelet aggregation in European Americans and African Americans [13, 15]. PEAR1 encodes a 1037 amino acid platelet cell surface receptor and, upon activation, an intracellular tyrosine residue is phosphorylated, followed by degranulation, amplification of the glycoprotein IIb/IIIa pathway and sustained platelet aggregation, most likely through the PI3K/Akt pathway [21, 22]. We now extend the association to include collagen-mediated platelet aggregation, findings confirmed in an independent group of African Americans. The variant identified by rs12041331 has been shown to regulate expression of PEAR1 protein in a dosedependent fashion [15]. Taken together, these data suggest 
that PEAR1 levels are important effectors of platelet aggregation in both African and European Americans.

Of the five novel variants associated with platelet aggregation in the discovery sample, 2 were confirmed in at least one of our replication cohorts. Three novel variants did not replicate, suggesting they might be false positives. The most intriguing replicated variant was rs11202221 in BMPR1A; the G-allele of this SNP was associated with decreased ADP-induced platelet aggregation. This is of interest because BMPR1A has been implicated in vascular calcification as well as in the development of atherosclerosis [23], and platelets play a role in pathogenesis of the latter. BMPR1A encompasses $168 \mathrm{~kb}$ in the 10q23.2 region and encodes a 532 amino acid long single-pass cell surface receptor. This receptor belongs to the BMP receptor family of the transforming growth factor-beta (TGF- $\beta$ ) receptor superfamily and is expressed widely in various tissues. On ligand binding, BMPR1A activates intracellular signaling pathways, commonly leading to altered gene expression. Although BMPR1A has not been identified in platelets, several reports indicate it is expressed in megakaryocytes [24, 25], the bone marrow progenitor cell that produces platelets. Thus, the variants in BMPR1A that are associated with platelet aggregation could alter BMPR1A expression and/or function in megakaryocytes, which in turn could alter gene expression in signaling molecules mediating ADP-induced platelet aggregation. Alternatively, these BMPR1A SNPs could be in LD with other causative SNPs in either protein-coding or non-protein coding genes. Among the protein-coding genes near BMPR1A, transcripts of MMRN2, GLUD1, WAPL, and PAPSS2 are present in platelets, however, only GLUD1 (glutamate dehydrogenase 1) and PAPSS2 (3'-phosphoadenosine 5 -phosphosulfate synthase 2) protein-products are present in platelets [26-28]. There are no microRNAs or lincRNAs within $500 \mathrm{~kb}$ of rs11202221.

It is intriguing that rs11202221 in BMPR1A did not replicate in European Americans. It is unlikely that this SNP is a false positive because 1) it replicated in PGAP African Americans, 2) the effect of the minor allele on platelet aggregation was the same direction in the two African American populations, and 3) there were 5 other SNPs in BMPR1A that showed association $\left(\mathrm{p}<10^{-5}\right)$ with ADP-induced platelet aggregation in both African American populations. LD patterns differ dramatically between European (CEU) and African (YRI) populations represented in the 1000 Genomes Project (see Additional file 1 Fig. S3). There is a $53 \mathrm{~Kb}$ block of LD including rs11202221 in the CEU reference population where variants are high in frequency (G allele frequency $20 \%$ at rs11202221, and MAF of most SNPs in the block are $\sim 20 \%$ ). In contrast, no LD block in the YRI reference population includes rs11202221 and the allele frequency of the $\mathrm{G}$ allele is considerably lower (4\%). Given the tagging approach employed herein with the GWAS array, we speculate that rs11202221 tags the true 'causal' variant which itself may be low in frequency. Under this hypothesis, the 'causal' variant would consequently be tagged with higher correlation in the African American population in contrast to the European American population and therefore yield significant results in the African Americans but not European Americans. Future work will involve a targeted resequencing in the full dataset to fully examine all sequence-identified variants in this region and specifically test this hypothesis.

The second novel association with ADP-mediated aggregation is of an intergenic variant at $18 \mathrm{q} 22.3$ locus with ADP-mediated platelet aggregation. The $500 \mathrm{~kb}$ region on either side of this variant contains a few genes, but the transcript of only one, FBXO15 (which encodes F-box protein 15), has been reported at low levels in platelets. F-box proteins are important in substrate recognition by certain ubiquitin protein ligase complexes and thus are important in regulating protein degradation $[29,30]$. While it is possible that this variant may affect protein degradation in platelets, the role of FBXO15 in platelet aggregation remains unexplored.

Different agonists and agonist concentrations were utilized to generate more refined platelet signaling pathway phenotypes. Collagen activates platelets via glycoprotein VI, which signals via an immunoreceptor tyrosine activation motif [31]. ADP induces platelet activation through the P2 $\mathrm{Y}_{12}$ and P2Y 1 G protein coupled receptors (GPCRs), while epinephrine activates platelets via a different GPCR, the $\alpha 2 \mathrm{~A}$-adrenergic receptor [32]. Each of these GPCRs activates different $G$ protein families, which in turn activate different sets of signaling molecules. Eventually, these different proximal signaling pathways converge to a final common pathway resulting in integrin $\alpha \operatorname{IIIb} \beta 3$ activation and platelet aggregation. Because SNPs in PEAR1 were associated with platelet aggregation induced by all three of these physiologic agonists, our data suggest a potential role for PEAR1 in a shared signaling pathway downstream of receptor-proximal signaling. Furthermore, ADP at low concentrations (e.g., $2 \mu \mathrm{M}$ ) induces rapid and reversible platelet aggregation through $\mathrm{G}_{\mathrm{q}}$-coupled $\mathrm{P} 2 \mathrm{Y}_{1}$, whereas high ADP concentrations (e.g., $10 \mu \mathrm{M}$ ) induce $\mathrm{G}_{\mathrm{i}}$-coupled $\mathrm{P} \mathrm{Y}_{12}$ inhibition of adenyl cyclase and complete aggregation [33]. Thus, our findings that different loci were associated with different concentrations of ADP-induced platelet aggregation support hypotheses whereby ALDH1L1-AS2, SUFU and BMPR1A regulate primarily platelet $\mathrm{G}_{\mathrm{q}}$ signaling and $S D K 2$ regulates primarily $\mathrm{G}_{\mathrm{i}}$ signaling in platelets.

\section{Conclusions}

In conclusion, we report here results of the first GWAS of agonist-induced platelet aggregation in African Americans. Our results confirm the importance of PEAR1 in platelet 
biology and establish that variants in this gene affect platelet function in both African and European Americans. In addition, we have discovered and replicated novel loci associated with ADP-mediated platelet aggregation, one of which (rs11202221 in BMPR1A) may affect platelet function in African Americans but not in European Americans. Inhibition of ADP-induced platelet aggregation with thienopyridines is a mainstay of CAD treatment, and it will be important to consider race in the pharmacogenetics of these anti-platelet therapies. Further study of the loci discovered in this report may provide important insights into platelet biology and may identify targets for the development of novel anti-platelet agents.

\section{Methods}

\section{Genetic Study of Atherosclerosis Risk (GeneSTAR) cohort}

The design of GeneSTAR has been reported previously $[10,34]$. Briefly, African American and European American probands with documented early-onset CAD were identified at the time of the event at ten Baltimore hospitals. Their apparently healthy family members (siblings, offspring of probands and siblings, and parents of the offspring) were enrolled. Eligible participants were interviewed by a nurse practitioner and self-reported their age and race. They underwent a cardiovascular history and physical examination and assessment of cardiovascular risk factors. Individuals with personal history of CAD, bleeding disorders, serious comorbidities, or who were taking anticoagulants, antiplatelet agents or nonsteriodal anti-inflammatory agents that could not be safely discontinued for two weeks prior to the study start were excluded. Individuals were also excluded if they had abnormal platelet count $(<100,000 / \mu \mathrm{L}$ or $>500,000 / \mu \mathrm{L})$ hematocrit $(<30 \%)$, or white blood cell count $(>20,000 / \mu \mathrm{L})$. The study was approved by the Johns Hopkins Institutional Review Board and all study participants gave informed consent (Additional file 1: Fig. S1).

\section{Platelet Genes and Physiology (PGAP) cohort}

Healthy volunteers were recruited between 2001-2006 in Houston, Texas. The study protocol was approved by the Institutional Review Boards of Baylor College of Medicine and Thomas Jefferson University, and informed consent was obtained from all participants. Subjects were 18-80 years of age and were excluded if they had diabetes or hypertension, or had taken anti-platelet drugs within the past 10 days, anti-inflammatory drugs within the past $48 \mathrm{~h}$, more than one prescribed medication (excluding oral contraceptives and hormone replacement therapy), or had been exposed to medication that affected the bone marrow. Eligible participants were interviewed by a study coordinator and self-reported their age and race. Hematocrit and platelet counts were in the normal range.

\section{Platelet aggregation studies}

In both cohorts, blood was obtained by venipuncture and collected into EDTA (for complete blood cell counts) or $3.2 \%$ sodium citrate (for platelet function testing). Platelet counts were determined by automated cell counter. Platelet-rich plasma was prepared from whole blood by centrifugation at $180 \times \mathrm{g}$ for $15 \mathrm{~min}$, and platelet-poor plasma was prepared by centrifugation at $2000 \mathrm{x} g$ for $10 \mathrm{~min}$. Platelet counts were adjusted to $200,000 / \mu \mathrm{L}$ by diluting platelet-rich plasma with platelet-poor plasma.

Optical aggregometry with platelet-rich plasma was used to measure platelet aggregation (0-100 \%) and maximal aggregation was determined within $5 \mathrm{~min}$ of stimulating samples with various platelet agonists. In GeneSTAR, platelets were stimulated with adenosine diphosphate (ADP 2 and $10 \mu \mathrm{M})$, arachidonic acid $(1.6 \mathrm{mM})$, collagen $(2 \mu \mathrm{g} / \mathrm{mL})$ or epinephrine $(2 \mu \mathrm{M})$. In PGAP, platelets were stimulated with ADP $(4 \mu \mathrm{M})$, collagen $(2 \mu \mathrm{g} / \mathrm{mL})$, arachidonic acid $(1.6 \mathrm{mM})$ and epinephrine $(1.5 \mu \mathrm{M})$. As agonist doses were not identical for ADP and epinephrine in GeneSTAR and PGAP, we compared the platelet aggregation results with both doses of ADP from GeneSTAR with ADP $4 \mu \mathrm{M}$ from PGAP and epinephrine $2 \mathrm{uM}$ from GeneSTAR with epinephrine $1.5 \mu \mathrm{M}$ from PGAP analyses.

\section{Genotype data and quality control}

In GeneSTAR, genome-wide SNP genotyping was performed at deCODE Genetics, Inc. using the Human $1 \mathrm{Mv1}$ 1_C array from Illumina, Inc. with an average call rate per sample of $99.65 \%$ (overall missing data rate = $0.35 \%$ ). Using 25 duplicate pairs, the reproducibility rate was $>99.95 \%$. Samples that showed Mendelian errors > $5 \%$ were excluded. We also excluded SNPs with call rate $<90 \%$, MAF $<5 \%$ and/or severe deviation from Hardy Weinberg equilibrium ( $p$-value $<10^{-6}$ ) in the discovery sample.

In PGAP, genotyping was performed using the Illumina Human1M BeadChip at Baylor College of Medicine. Individuals with greater than $3 \%$ missing genotypes, or average heterozygosity greater than 2 standard deviations from the mean were excluded. Any SNP locus with $>5 \%$ missing genotypes or deviation from Hardy Weinberg Equilibrium ( $\mathrm{p}$-value $<10^{-6}$ ) was removed. In the final analysis, only those SNPs were included in the analysis for which data were available in both the discovery and replication samples.

\section{Data analysis}

Analyses were performed separately for the African Americans and European Americans in GeneSTAR. All variables were assessed for Gaussian distribution. Since the distribution response to epinephrine and collagen in both African American cohorts was bimodal, we dichotomized the phenotypes at the visual intersection of the 
two modal distributions. For all platelet aggregation phenotypes, we evaluated age- and sex-adjusted linear additive models. To account for intrafamilial relationships in GeneSTAR, linear mixed effects models were used to test the association under an additive model between a SNP and specific phenotype adjusted for age, sex and 2 principal components [13]. In our previous work with platelet aggregation phenotypes, we have found that only the first two principal components are associated with platelet phenotypes and that including more than two principal components in GWAS analyses does not provide additional information. The formulation of the mixed model follows in a matrix form: $\mathrm{Y}=\mathrm{XB}+\mathrm{ZU}+\varepsilon$, where $Y$ is an $m \times 1$ vector of responses; $X$ is an $m \times p$ design matrix of the fixed effects; $B$ is the parameter $p \times$ 1 vector of fixed effects; $\mathrm{Z}$ is an $\mathrm{m} \times \mathrm{q}$ incidence matrix of random effects, and $U$ is a $q \times 1$ vector of random effects with $E(U)=0$, and covariance matrix $G ; 0$ is an $\mathrm{m} \times 1$ vector of random effects with $\mathrm{E}(0)=0$ and covariance matrix R. We tested whether the SNPs additive effects are different from zero, and especially we identified the highest significances. These models were run using PROC MIXED in SAS (v. 9.1.3 for Linux OS) with the option for EMPIRICAL variance and including the family identification number in the random effects to account for relatedness $[35,36]$ (SAS Institute, Cary, North Carolina, 1996). For collagen $2 \mu \mathrm{g} / \mathrm{mL}$ phenotypes, logistic models were used using generalized estimating equations for GeneSTAR. The data from PGAP was analyzed under additive models using $\mathrm{R}$ library GenABEL [37]. We adjusted for cryptic population structure using the method proposed by Chen and Abecasis as implemented in the "mmscore' function in the GenABEL package [38]. The mmscore function uses the formula $\left\{\left((G-E[G]) \mathrm{V}^{-1} \times \mathrm{Y}\right)^{2}\right\}$ / $\left\{(G-E[G]) V^{-1}(G-E[G])\right\}$ where $G$ is the vector of genotypes (coded $0,1,2$ ) and $E[G]$ is a vector of (strataspecific) mean genotypic values; $\mathrm{V}^{-1}$ is the inverse of the variance-covariance matrix at the maximum of polygenic model and $\mathrm{Y}$ are residuals after both the effect of covariates and the estimated polygenic effect (breeding values) are factored out [37]. We also used linear or logistic regression adjusting for age, sex, and 2 principal components and found similar results; hence we are presenting results obtained using mmscore function. In all three cohorts, we tested whether the SNP additive effects differed from zero. GWAS significance threshold was set at $\mathrm{p}$-value $<5 \times 10^{-8}$ and for replication, a Bonferroni adjusted p-value of $<0.008$ was considered significant. Suggestive association between a variant and a phenotype was said to be present if association p-value was $<5 \times 10^{-6}$. The African American cohort of the GeneSTAR study served as the discovery sample and the PGAP cohort served as a replication sample. As the PGAP sample size was small and hence had limited power, we also examined the European American cohort of the GeneSTAR as an additional replication sample. Due to the differences in the linkage disequilibrium pattern between populations of African and European descent, we also examined the association of the phenotype with nearby SNPs (within $250 \mathrm{kbp}$ on either side) in the European American replication sample if the lead SNP in the African American discovery sample was not statistically significant in the African American or European American replication cohorts.

\section{Availability of supporting data}

Based on participant consent forms, only aggregate data are available on dbGAP. Individual level data will be available using a Limited Access Agreement, which requires submission of an application and a study description to the GeneSTAR Study Steering Committee at The Johns Hopkins University. All data and all information will be fully deidentified according to our consent process. For further information, please visit: http://www.genestarstudy. com/For-Researchers.html.

\section{Additional file}

Additional file 1: The supplemental material contains the following:
Table S1. Hemostatic characteristics across genotypes of replicated SNPS.
Table S2. Loci with suggestive association findings in the GWAS of
epinephrine-mediated platelet aggregation in African Americans. Table S3.
Loci with suggestive association findings in the GWAS of collagen-mediated
platelet aggregation in African Americans. Table S4. ADP-mediated Platelet
Aggregation across Genotypes of the Two Novel Genetic Variants. Table S5.
Loci with suggestive association findings in the GWAS of ADP $2 \mu$ M-mediated
platelet aggregation in African Americans. Table S6. Loci with suggestive
association findings in the GWAS of ADP 10 MM-mediated platelet
aggregation in African Americans. Table S7. Loci with suggestive
association findings in the GWAS of arachidonic acid-mediated platelet
aggregation in African Americans. Figure S1. Study Design of the Genetic
Study of Aspirin Responsiveness (GeneSTAR) and Platelet Genetics and
Physiology (PGAP). Figure S2. Quantile-Quantile (QQ) plots with genomic
inflation factors ( $\lambda$ ). Figure S3. Linkage disequilibrium plots of European
descent population (CEU) and African descent population (YRI) based on
the data from 1000 Genomes Project.

\section{Abbreviations}

GWAS: Genome-wide association study; PGAP: Platelet genes and physiology; EDTA: Ethylenediaminetetraacetic acid; GeneSTAR: Genetic Study of Atherosclerosis Risk; SNP: single nucleotide polymorphisms; PEAR1: Platelet endothelial aggregation receptor 1; ADP: Adenosine diphosphate; ALDH1L1-AS2: Aldehyde dehydrogenase 1 family, member L1-antisense RNA 2; SUFU: Suppressor of fused homologue; BMPR1A: Bone morphogenetic protein receptor, type IA; SDK2: Sidekick cell adhesion molecule 2; FRMPD1: FERM and PDZ domain containing 1; CAD: coronary artery disease; MAF: Minor allele frequency; PI3K/Akt: phosphatidylinositol 3-kinase/protein kinase B; BMP: Bone morphogenetic protein; TGF- $\beta$ : Transforming growth factor-beta; MMRN2: Multimerin 2; GLUD1: glutamate dehydrogenase 1; WAPL: Wings apart-like homolog; PAPSS2: 3'-phosphoadenosine 5'-phosphosulfate synthase 2; lincRNA: Long intervening noncoding RNA; SLC41A3: Solute carrier family 41, member 3; FBXO15: F-box protein 15.

\section{Competing interests}

The authors declare that they have no competing interests. 


\section{Author's contributions}

LCB, DMB, NF, LRY conceived and designed the GeneSTAR cohort. PFB, SML, CS conceived and designed the PGAP cohort. RQ conceived the idea for the current study. RQ, LRY, BS, and SML performed analysis and interpreted results. $L C B, D M B, P F B, N F$, and $R M$ gave critical input in the design and analysis of the current study and interpretation of results. $R Q$ wrote the first draft and LCB, DMB, PFB, SML, NF, LRY, RM, and BS helped in draft preparation and provided critical input in manuscript completion. All authors read and approved the final manuscript.

\section{Acknowledgements}

The GeneSTAR study was supported by the National Heart, Lung, and Blood Institute (NHLBI) through the PROGENI (U01 HL72518) and STAMPEED (R01 HL087698-01). Dr. Rehan Qayyum was supported by NIH grant K23HL105897 and Dr. Paul F. Bray was supported by NIH grant HL88458. Funding source had no role in the design of study, analysis of data, interpretation of results or preparation of manuscript.

\section{Author details}

${ }^{1}$ The GeneSTAR Research Center, Department of Medicine, Johns Hopkins University School of Medicine, Baltimore, Maryland 21287, USA. ${ }^{2}$ Department of Medicine, University of Tennessee College of Medicine, Chattanooga, Tennessee 37403, USA. ${ }^{3}$ Department of Anesthesiology and Critical Care Medicine, Johns Hopkins University School of Medicine, Baltimore, Maryland 21287, USA. ${ }^{4}$ Molecular and Human Genetics, Baylor College of Medicine, Houston, Texas 77030, USA. ${ }^{5}$ Integrative Computational BioScience Center, Department of Biochemistry, Faculty of Medicine, Siriraj Hospital, Mahidol University, Bangkok, 10700, Thailand. 'Department of Medicine, Thomas Jefferson University, Jefferson Medical College, Philadelphia, Pennsylvania 19107, USA.

\section{Received: 5 February 2015 Accepted: 13 May 2015} Published online: 30 May 2015

\section{References}

1. Nieswandt B, Pleines I, Bender M. Platelet adhesion and activation mechanisms in arterial thrombosis and ischaemic stroke. J Thromb Haemost. 2011;9 Suppl 1:92-104.

2. Lievens D, von Hundelshausen P. Platelets in atherosclerosis. Thromb Haemost. 2011;106(5):827-38

3. Yee DL, Sun CW, Bergeron AL, Dong JF, Bray PF. Aggregometry detects platelet hyperreactivity in healthy individuals. Blood. 2005;106(8):2723-9.

4. Marcucci R, Gori AM, Paniccia R, Giusti B, Valente S, Giglioli C, et al. High on-treatment platelet reactivity by more than one agonist predicts 12-month follow-up cardiovascular death and non-fatal myocardial infarction in acute coronary syndrome patients receiving coronary stenting. Thromb Haemost. 2010;104(2):279-86.

5. Breet NJ, van Werkum JW, Bouman HJ, Kelder JC, Ruven HJ, Bal ET, et al. Comparison of platelet function tests in predicting clinical outcome in patients undergoing coronary stent implantation. JAMA 2010;303(8):754-62

6. Bordeaux BC, Qayyum R, Yanek LR, Vaidya D, Becker LC, Faraday N, et al. Effect of obesity on platelet reactivity and response to low-dose aspirin. Prev Cardiol. 2010;13(2):56-62

7. Faraday N, Yanek LR, Vaidya D, Kral B, Qayyum R, Herrera-Galeano JE, et al. Leukocyte count is associated with increased platelet reactivity and diminished response to aspirin in healthy individuals with a family history of coronary artery disease. Thromb Res. 2009;124(3):311-7.

8. Vaidya D, Yanek LR, Faraday N, Moy TF, Becker LC, Becker DM. Native platelet aggregation and response to aspirin in persons with the metabolic syndrome and its components. Metab Syndr Relat Disord. 2009;7(4):289-96.

9. Bordeaux B, Yanek LR, Moy TF, White LW, Becker LC, Faraday N, et al. Casual chocolate consumption and inhibition of platelet function. Prev Cardiol. 2007;10(4):175-80.

10. Becker DM, Segal J, Vaidya D, Yanek LR, Herrera-Galeano JE, Bray PF, et al. Sex differences in platelet reactivity and response to low-dose aspirin therapy. JAMA. 2006:295(12):1420-7.

11. Bray PF, Mathias RA, Faraday N, Yanek LR, Fallin MD, Herrera-Galeano JE, et al. Heritability of platelet function in families with premature coronary artery disease. J Thromb Haemost. 2007;5(8):1617-23.
12. Kunicki TJ, Nugent DJ. The genetics of normal platelet reactivity. Blood. 2010;116(15):2627-34.

13. Johnson $A D$, Yanek LR, Chen MH, Faraday $N$, Larson MG, Tofler $G$, et al. Genome-wide meta-analyses identifies seven loci associated with platelet aggregation in response to agonists. Nat Genet. 2010;42(7):608-13

14. Casto AM, Feldman MW. Genome-wide association study SNPs in the human genome diversity project populations: does selection affect unlinked SNPs with shared trait associations? PLoS Genet. 2011;7(1), e1001266.

15. Faraday N, Yanek LR, Yang XP, Mathias R, Herrera-Galeano JE, Suktitipat B, et al. Identification of a specific intronic PEAR1 gene variant associated with greater platelet aggregability and protein expression. Blood. 2011;118(12):3367-75.

16. Thomas KL, Honeycutt E, Shaw LK, Peterson ED. Racial differences in longterm survival among patients with coronary artery disease. Am Heart J. 2010;160(4):744-51.

17. Berry JD, Dyer A, Cai X, Garside DB, Ning H, Thomas A, et al. Lifetime risks of cardiovascular disease. N Engl J Med. 2012;366(4):321-9.

18. Marenberg ME, Risch N, Berkman LF, Floderus B, de Faire U. Genetic susceptibility to death from coronary heart disease in a study of twins. N Engl J Med. 1994;330(15):1041-6.

19. Rissanen AM. Familial occurrence of coronary heart disease: effect of age at diagnosis. Am J Cardiol. 1979;44(1):60-6.

20. Rissanen AM. Familial aggregation of coronary heart disease in a high incidence area (North Karelia, Finland). Br Heart J. 1979;42(3):294-303.

21. Nanda N, Bao M, Lin H, Clauser K, Komuves L, Quertermous T, et al. Platelet endothelial aggregation receptor 1 (PEAR1), a novel epidermal growth factor repeat-containing transmembrane receptor, participates in platelet contact-induced activation. J Biol Chem. 2005;280(26): 24680-9.

22. Kauskot A, Di Michele M, Loyen S, Freson K, Verhamme P, Hoylaerts MF. A novel mechanism of sustained platelet alphallbbeta3 activation via PEAR1. Blood. 2012;119(17):4056-65.

23. Derwall M, Malhotra R, Lai CS, Beppu Y, Aikawa E, Seehra JS, et al. Inhibition of bone morphogenetic protein signaling reduces vascular calcification and atherosclerosis. Arterioscler Thromb Vasc Biol. 2012; 32(3):613-22

24. Garimella R, Kacena MA, Tague SE, Wang J, Horowitz MC, Anderson HC. Expression of bone morphogenetic proteins and their receptors in the bone marrow megakaryocytes of GATA-1(low) mice: a possible role in osteosclerosis. J Histochem Cytochem. 2007;55(7):745-52.

25. Watkins NA, Gusnanto A, de Bono B, De S, Miranda-Saavedra D, Hardie DL, et al. A HaemAtlas: characterizing gene expression in differentiated human blood cells. Blood. 2009:113(19):e1-9.

26. Burkhart JM, Vaudel M, Gambaryan S, Radau S, Walter U, Martens L, et al. The first comprehensive and quantitative analysis of human platelet protein composition allows the comparative analysis of structural and functional pathways. Blood. 2012;120(15):e73-82

27. Rowley JW, Oler AJ, Tolley ND, Hunter BN, Low EN, Nix DA, et al. Genomewide RNA-seq analysis of human and mouse platelet transcriptomes. Blood. 2011;118(14):e101-11.

28. Bugert $\mathrm{P}$, Kluter $\mathrm{H}$. Profiling of gene transcripts in human platelets: an update of the platelet transcriptome. Platelets. 2006;17(7):503-4.

29. Kato M, Kito K, Ota K, Ito T. Remodeling of the SCF complex-mediated ubiquitination system by compositional alteration of incorporated F-box proteins. Proteomics. 2010;10(1):115-23.

30. Ho MS, Tsai PI, Chien CT. F-box proteins: the key to protein degradation. J Biomed Sci. 2006;13(2):181-91.

31. Watson SP, Gibbins J. Collagen receptor signalling in platelets: extending the role of the ITAM. Immunol Today. 1998;19(6):260-4.

32. Smyth SS, Woulfe DS, Weitz II, Gachet C, Conley PB, Goodman SG, et al. G-protein-coupled receptors as signaling targets for antiplatelet therapy. Arterioscler Thromb Vasc Biol. 2009;29(4):449-57.

33. Jin J, Kunapuli SP. Coactivation of two different G protein-coupled receptors is essential for ADP-induced platelet aggregation. Proc Natl Acad Sci U S A. 1998;95(14):8070-4.

34. Qayyum R, Becker DM, Yanek LR, Moy TF, Becker LC, Faraday N, et al. Platelet inhibition by aspirin 81 and 325 mg/day in men versus women without clinically apparent cardiovascular disease. Am J Cardiol. 2008; 101(9):1359-63 
35. Littell RC, Milliken GA, Stroup WW, Wolfinger RD. SAS ${ }^{\oplus}$ for Mixed Models. 2nd ed. Cary: SAS Institute; 2006.

36. Suktitipat B, Mathias RA, Vaidya D, Yanek LR, Young JH, Becker LC, et al. The robustness of generalized estimating equations for association tests in extended family data. Hum Hered. 2012;74(1):17-26.

37. Aulchenko YS, Ripke S, Isaacs A, van Duijn CM. GenABEL: an R library for genome-wide association analysis. Bioinformatics. 2007;23(10):1294-6.

38. Chen WM, Abecasis GR. Family-based association tests for genomewide association scans. Am J Hum Genet. 2007;81(5):913-26.

Submit your next manuscript to BioMed Central and take full advantage of:

- Convenient online submission

- Thorough peer review

- No space constraints or color figure charges

- Immediate publication on acceptance

- Inclusion in PubMed, CAS, Scopus and Google Scholar

- Research which is freely available for redistribution 